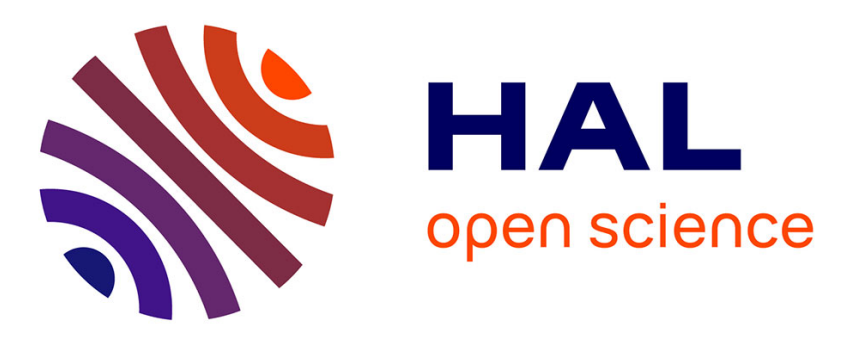

\title{
Gespräch über Probleme der epigraphischen Forschung in Frankreich
}

\author{
Robert Favreau, Raymund Kottje
}

\section{To cite this version:}

Robert Favreau, Raymund Kottje. Gespräch über Probleme der epigraphischen Forschung in Frankreich. Helga Giersiepen, Raymund Kotje. Inschriften bis 1300, Probleme und Aufgaben ihrer Erferschung, 94, Westdeutscher Verlag, pp.85-102, 1995, Abhandlungen der Nordrhein-Westfälischen Akademie der Wissenschaften. halshs-03326780

\section{HAL Id: halshs-03326780 \\ https://shs.hal.science/halshs-03326780}

Submitted on 26 Aug 2021

HAL is a multi-disciplinary open access archive for the deposit and dissemination of scientific research documents, whether they are published or not. The documents may come from teaching and research institutions in France or abroad, or from public or private research centers.
L'archive ouverte pluridisciplinaire HAL, est destinée au dépôt et à la diffusion de documents scientifiques de niveau recherche, publiés ou non, émanant des établissements d'enseignement et de recherche français ou étrangers, des laboratoires publics ou privés. 


\title{
GESPRÄCH ÜBER PROBLEME DER EPIGRAPHISCHEN FORSCHUNG IN FRANKREICH
}

\begin{abstract}
Um einen gezielten Einblick in Stand und Probleme der epigraphischen Arbeit in Frankreich zu gewinnen, wurden von Prof. KotTJE Fragen gestellt, die Prof. FAVReAu je unmittelbar beantwortete. Die Übersetzung hat Herr BAYER übernommen. Fragen und Antworten werden im folgenden zusammenhängend präsentiert - die Antworten in erweiterter Fassung.
\end{abstract}

a) Fragen zur Bearbeitung der mittelalterlichen Inschriften Frankreichs :

Auf der Epigraphik-Fachtagung in Landshut haben Sie, Herr Favreau, 1980 über das Projekt eines Corpus der mittelalterlichen Inschriften Frankreichs berichtet, das Sie 1969 begonnen haben und seitdem mit einer kleinen Forscher- gruppe in Poitiers bearbeiten ${ }^{1}$. Die zeitlichen Grenzen dieses Corpus sind einerseits vorgegeben durch die von anderen unternommene Sammlung der Inschriften Galliens bis zur Karolingerzeit bis etwa 750, andererseits wegen der Materialfülle, also aus pragmatischen Gründen, auf 1300 als allerdings nicht starren Endpunkt festgelegt worden. Im Hinblick auf das Rahmenthema unserer Tagung würde heute interessieren, ob sich für diese Begrenzung auch inhaltliche und/oder formale Kriterien anführen lassen, vor allem für den Endpunkt 1300. Daher meine Fragen :

1) Gibt es eine Entwicklung der Schrift der Inschriften Frankreichs, die um 1300 bzw. im 13./14. Jahrhundert einen irgendwie auffallenden Wandel erkennen laßt ? Gibt es einen solchen Wandel etwa im Dekor, in den Formen der Schrift oder ihres Umfelds?

2) Sind regionale Unterschiede festzustellen, zwischen Nord und Süd oder gewissen Räumen mit eigenständiger kultureller Tradition ? Sind etwa Inschriften auf Stein oder Holz oder Metall unterschiedlich verarbeitet? Ergeben sich von daher Indizien für einen Wandel um 1300 ?

3) Können sprachliche Gesichtspunkte angeführt werden, etwa des Übergangs vom Lateinischen zur Volkssprache oder der Verwendung von Metren oder Reim ?

4) Ist ein Wandel in der Form der Datierung erfolgt ?

5) Kann man von einem Wandel im Inhalt der Inschriften sprechen und damit vielleicht auch von einem Wandel des Interesses an der Herstellung von Inschriften ?

Le Corpus des inscriptions de la France Médiévale sera abrégé en C.I.F.M.

${ }^{1}$ Vgl. R. Favreau, Le corpus des inscriptions de la France médiévale, in: R. M. Kloos (Hrsg.), Fachtagung für lateinische Epigraphik des Mittelalters und der Neuzeit - Landshut, 18.-20. Juli 1980 (Münchener Historische Studien, Abt. Geschichtl. Hilfswissenschaften, Bd. 19), Kallmünz 1982, S. 6169 (dt. Zusammenfassung S. 69-72). Vgl. auch R. Favreau, Les inscriptions médiévales (Typologie des sources du moyen Âge occidental 35), Turnhout 1979, S. 112-117. 
Wir wären Ihnen für einige Hinweise dankbar.

b) Inscriptions médiévales antérieures à 1300. Réflexions à propos de l'expérience française

Le Congrès d'épigraphie du Moyen Âge et du début des temps modernes réuni à Bonn du 3 au 5 juin 1993 avait pour thème : les inscriptions antérieures à 1300, et $M$. le professeur Raymund Kottje m'avait demandé d'apporter des réponses à une série de questions concernant l'expérience française, Je m'inspirerai donc de ces questions pour examiner, à partir de la recherche menée à Poitiers, la problématique propre aux inscriptions de cette période chronologique.

L'équipe chargée de recenser, étudier et publier les inscriptions de la France médiévale travaille, dans un premier temps, sur les textes compris entre les débuts de l'époque carolingienne (vers le milieu du $\mathrm{VIII}^{\mathrm{e}}$ siècle) et la fin du XIII ${ }^{\mathrm{e}}$ siècle. Pourquoi ? La réponse est évidente pour le terminus a quo : les inscriptions antérieure au VIII ${ }^{\mathrm{e}}$ siècle ont été publiées par Edmont Le Blant au XIX siècle $^{2}$ et le travail de Le Blant est en cours de refonte, mise à jour, approfondissement par une équipe, créée en 1965 à l'initiative du professeur Henri-Irénée Marrou, et qui a déjà publié les volumes consacrés à la province de Trèves et à la Viennoise du Nord ${ }^{3}$. La demande de création d'une équipe qui aurait en charge l'étude des inscriptions postérieures au champ chronologique retenu par Le Blant a été présentée au C.N.R.S. en 1968 par le professeur Edmond-René Labande, alors directeur du Centre d'Études Supérieures de Civilisation Médiévale, comme un prolongement de la photothèque d'art roman du Centre, où continuellement étaient engrangées des photographies de monuments ou objets accompagnés d'inscriptions. Le C.E.S.C.M. avait été crééen 1953 pour l'étude de la période romane, remarquablement représentée dans les monuments de la ville et de la région. C'est donc tout naturellement pour cette même période qu'a été formulée la demande. Le C.N.R.S. a ainsi créé en 1969, une équipe chargée des inscriptions médiévales françaises entre le milieu du VIII ${ }^{\mathrm{e}}$ siècle et 1250. Dans la pratique le terminus ante quem a été repoussé à 1300. Ces limites chronologiques ont l'avantage de permettre de réaliser le travail dans un temps défini. Sur le plan scientifique elles présentent évidemment des difficultés, comme toute limite chronologique en elle-même, interrompant arbitrairement une évolution continue. Il est clair que la périodisation retenue peut convenir à la France, mais ne pourrait pas nécessairement être adaptée pour d'autres pays.

\section{L'écriture}

La première question posée est celle de l'évolution de l'écriture. Question que ne peuvent pas écarter les épigraphistes, même si on peut estimer, avec Jean Mallon, que pour eux la préoccupation paléographique n'est que secondaire par rapport à l'étude du texte ${ }^{4}$.

L'étude de l'évolution de l'écriture épigraphique doit constituer un des chapitres del'histoire générale de la paléographie, c'est-à-dire d'un des faits majeurs de civilisation qu'est l'écrit. L'écriture des inscriptions peut aussi apporter une contribution à la datation des textes. Le Blant estimait que «demander à la forme. des lettres des éléments d'appréciation certains au point de vue chronologique serait chose hasardeuse », car on trouve souvent « sur un même marbre, parfois dans le même vocable un même mot sous des formes très diverses que l'on pourrait croire d'époques différentes. Ce n'est pas à dire qu'il y ait lieu de négliger certaines données de la paléographie ». Ainsi de « toute une série de lettres dont la haste dépasse les membres latéraux, [qui] ne paraît guère

\footnotetext{
${ }^{2}$ Edmond Le Blant, Inscriptions chrétiennes de la Gaule antérieures au VII siècle, Paris, 1856 et 1865, 2 vol.; Nouveau recueil des inscriptions chrétiennes de la Gaule antérieures au VIII siècle, Paris, 1892.

${ }^{3}$ Nancy Gauthier, Recueil des inscriptions chrétiennes de la Gaule antérieures à la Renaissance carolingienne, dir. H.-I. Marrou, I, Première Belgique, Paris 1975 ; Françoise Descombes, Recueil des inscriptions chrétiennes de la Gaule antérieures à la Renaissance carolingienne, dir. H.-I. Marrou, XV, Viennoise du Nord, Paris, 1985 ; Charles Pietri, «L'épigraphie chrétienne », Actes du colloque international du centenaire de L'Année épigraphique, 1988, p. 331-336.

${ }^{4}$ Jean Mallon, Paléographie romaine, Madrid, 1952, p. 55.
} 
qu'au commencement du VI ${ }^{\mathrm{e}}$ siècle $»^{5}$. Felice Grossi Gondi étudiant 83 inscriptions du $\mathrm{IX}^{\mathrm{e}}$ siècle, principalement romaines, conclut que le critère paléographique est, pour cette époque bien « incertain ${ }^{6}$. Historiens de l'art, Robert de Lasteyrie ${ }^{7}$ et Paul Deschamps ont utilisé leur connaissance de l'écriture épigraphique comme un élément d' appréciation sur la chronologie des monuments, en particulier du XII ${ }^{\mathrm{e}}$ siècle où, pour l'art, l'évolution est « constante et très rapide $»^{8}$. La paléographie des inscriptions peut aussi aider à localiser les objets sur lesquels elles ont été gravées, à mettre en relief des différences régionales. Cet aspect n'a guère retenu Paul Deschamps, qui voit une évolution générale de l'écriture épigraphique, en constatant toutefois des caractères particuliers pour les inscriptions de Rome du XII ${ }^{\mathrm{e}}$ siècle $^{9}$. Nicolette Gray au contraire s'est attachée à montrer, dans les inscriptions des $\mathrm{VIII}^{\mathrm{e}}, \mathrm{IX}^{\mathrm{e}}$ et $\mathrm{X}^{\mathrm{e}}$ siècles en Italie, des différences d'écriture selon que l'on est à Rome, dans l'Exarchat et le Pentapole, le royaume lombard, l'Italie du Sud ${ }^{10}$.

Histoire de l'écriture, datation, localisation, autant de points pour lesquels une étude paléographique est susceptible d'apporter une utile contribution, à condition de ne pas lui demander plus qu'elle ne peut offrir et de l'aborder d'une façon renouvelée. Je ferai ici cinq observations générales :

En premier lieu une étude de l'écriture doit partir d'un corpus photographique le plus complet possible. Pour intéressante - et même capitale - qu'elle soit, l'étude de Paul Deschamps est basée sur un trop petit nombre d'inscriptions pour qu'elle puisse être considérée comme sûre et définitive. Meyer Shapiro rendant compte de cette étude ${ }^{11}$ a, à juste titre, souligné qu'elle était appuyée sur trop peu de textes, une douzaine pour le $\mathrm{IX}^{\mathrm{e}}$ siècle, et d'un trop petit nombre de régions - ni l'Allemagne, ni l'Angleterre, fort peu l'Espagne et l'Italie, majoritairement la France du Sud-, pour qu'il soit possible d'en tirer des conclusions générales. Il est trop tôt pour pouvoir présenter de l'évolution générale de l'écriture épigraphique en Occident autre chose qu'une esquisse d'ensemble. Charles Perrat estimait même en 1955 que l'étude de Le Blant était « prématurée ${ }^{12}$, alors que ce dernier l'avait écrite après avoir dressé un corpus général des inscriptions chrétiennes de la Gaule et qu'il livrait les résultats d'une expérience d'une quarantaine d'années. Nous sommes encore loin de pouvoir présenter une histoire générale de l'écriture épigraphique au Moyen Âge. En ce qui concerne la France, le premier travail devra porter sur la France du Sud, pour laquelle on dispose d'un ensemble photographique aussi complet que possible, sans prétendre donner aux résultats de cette étude une valeur générale.

Le deuxième point est qu'il faut privilégier l'homogénéité. On ne peut mettre sur le même plan, dans la France du Sud, les inscriptions de Poitiers, de Toulouse, d'Elne, d'Arles, de Vienne, de Cluny ou de Saint-Benoît-sur-Loire. Il faut partir des ensembles les plus complets et les plus homogènes possibles avant de pouvoir présenter les traits communs d'évolution et les différences propres à chaque région. Peut-être trouvera-t-on même des différences à l'intérieur d'une même cité, d'où la nécessité d'une minutieuse étude de détail avant de proposer des conclusions. Un écueil du travail de P. Deschamps est d'avoir estimé que l'évolution des caractères des inscriptions comme se faisant «à peu près pareillement » en Languedoc, Poitou, Dauphiné, Provence, Bourgogne, aussi bien qu'en Asturies, Galice et Italie du Nord ${ }^{13}$.

\footnotetext{
${ }^{5}$ Edmond Le Blant, «Paléographie des inscriptions latines du $\mathrm{III}^{\mathrm{e}}$ siècle à la fin du $\mathrm{VII}^{\mathrm{e}} »$, Revue archéologique, 3e s., t. 29, 1896, p. 177-178.

${ }^{6}$ Felice Grossi Gondi, «Excursus sulla paleografia medievale epigrafica del sec. IX », Dissertazioni della Pontificia Accademia Romana di Archeologia, série Il, tomo XIII, 1918, p. 178.

${ }^{7}$ Robert de Lasteyrie, Études sur la sculpture française au moyen Âge, Paris, 1902, p. 48 (Monuments Piot, 8).

${ }^{8}$ Paul Deschamps, «Étude sur la paléographie des inscriptions lapidaires de la fin de l'époque mérovingienne aux dernières années du XII ${ }^{\mathrm{e}}$ siècle », Bulletin monumental, t. 88, 1929 (p. 5-81, 35 pl. h.-t.), p. 7.

${ }^{9}$ Ibid., p. 50.

${ }^{10}$ Nicolette Gray, «The Paleography of Latin Inscriptions in the Eigth, Ninth and Tenth Centuries in Italy », The Papers of the British School at Rome, XVI, 1948, p.38-171.

${ }^{11}$ Meyer Shapiro, The Art Bulletin, XII, n ${ }^{\circ}$ 1, 1930, p. 101-109.

${ }^{12}$ Charles Perrat, «Paléographie romaine », Relazioni nel X Congresso internazionale di Scienze storiche, Vol. I. Methodologia. Problemi generali Scienze ausiliare della storia, Florence, 1955, p. 356, n. 3.

${ }^{13}$ P. Deschamps, op. cit., p. 10.
} 
En troisième lieu on devra tenir compte avec attention du support. C'est bien ce qui a été fait de façon générale, aussi bien par Le Blant et Deschamps pour la France que par Grossi Gondi et N. Gray pour l'Italie, Bauer ${ }^{14}$ et Conrad ${ }^{15}$ pour l'Allemagne, Duran Gudiol pour l'Aragon ${ }^{16}$, chacun de ces auteurs partant des inscriptions lapidaires ou encore par W. Koch pour l'Autriche, pour une étude des inscriptions des peintures murales ${ }^{17}$. Or on est tenté, faute d'étude particulière, d'étendre aux textes écrits sur ardoise, sur métal, sur peinture murale, sur émail, sur vitrail, sur tapisserie, ce que l'on connaît de l'écriture sur pierre ou même de l'écriture des chartes en général. Siles parentés sont évidentes, il faudra des études en fonction des supports pour en mesurer la portée. Ceci est d'autant plus vrai qu'on ne possède pas, au point de vue de la paléographie, une étude particulière de la capitale ${ }^{18}$. L'écriture des inscriptions peintes me semble notamment pouvoir être un exemple d'une évolution propre, peut-être plus comparable à l'écriture des titres ou des légendes d'illustration dans les manuscrits qu'à l'écriture lapidaire.

Dans tous les cas, il faut faire une étude systématique et détaillée de chaque élément de l'écrit et de chaque lettre. Tous les auteurs ont souligné la complexité de l'étude : il faut étudier non seulement la forme des lettres mais le système d'abréviations, de contraction, de ponctuation, de réglure, indique Grossi Gondi ${ }^{19}$. Il faut prendre garde à l'aspect général, compter avec l'adresse plus ou moins grande de l'artiste, avec l'espace dont dispose le graveur, note Deschamps ${ }^{20}$ envisager aussi bien les formes que l'angle d'écriture, le ductus, le module, l'instrument, le support, la nature du texte, ajoute Mallon $^{21}$. Voici un certain nombre de points qui mériteraient, pour cette étude détaillée, d'être examinés :

- décor, occupation du champ épigraphique (marges, réglure, interligne) ;

- aspect général : espacement des mots, formes anguleuses, arrondies, étirées en hauteur, emploi de lettres plus petites que d'autres ;

- contraction du texte : conjonctions, enclavements, entrelacements, abréviations, forme des tildes d'abréviation;

- ponctuation, suscription du $O$ dans les dates ;

- lettres carrées, lettres onciales, lettres « fleuries », lettres perlées, lettres au trait redoublé ;

- et naturellement étude de chaque lettre.

Plutôt que de théoriser, il faut, humblement, minutieusement, interroger les textes, et dégager progressivement des termini a quibus et ante quos qui pourront, pour chaque région étudiée, apporter des bases plus silres pour les datations, les localisations, l'histoire de l'écriture. Voici quelques observations à partir d'une première étude des inscriptions de Vienne et de Toulouse. A Vienne la réglure comporte deux traits pour chaque ligne d'écriture en 1012 puis, de façon régulière, à partir de 1148. C'est aussi à partir du milieu du XII ${ }^{\mathrm{e}}$ siècle que les mots sont nettement séparés les uns des autres et que s'installe une ponctuation qui, au XIII ${ }^{\mathrm{e}}$ siècle, sera généralement un point médian entre chaque mot ; l'emploi d'une ponctuation par trois points verticaux est rare à Vienne (1186-1222). Les lettres carrées se raréfient au XII ${ }^{\mathrm{e}}$ siècle, disparaissent après 1202. On trouve dans les inscriptions de la ville un assez grand nombre de lettres fleuries de 1148 à 1285, des lettres perlées entre 1152 et le troisième quart du XII ${ }^{\mathrm{e}}$ siècle, des redoublements de trait dans les lettres entre 1152 et 1300 . Le développement des onciales se généralise dans la seconde moitié du XII ${ }^{\mathrm{e}}$ siècle, mais on ne trouve pas à Vienne, au XIII ${ }^{\mathrm{e}}$

\footnotetext{
${ }^{14}$ Konrad F. Bauer, « Mainzer Epigraphik. Beiträge zur Geschichte der mittelalterlichen Monumentalschrift », Zeitschrift des deutschen Vereins für Buchwesen und Schrifttum, 9e année, n 2-3, 1926, p. 1-45, 74 ill.

${ }^{15}$ Rudolf Conrad, NiederrheiniKhe Epigraphik vom achten bis zum dreizehnten Jahrhundert. Ein Beitrag zur Geschichte der monumentalen Schrift, Francfort-sur-le-Main, 1931, 70 p.

${ }^{16}$ Antonio Duran Gudiol, «Las inscripciones medievales de la provincia de Huesca », Estudios de edad media de la corona de Aragon, vol. VII, 1967, p. 3-19 (pour l'étude paléographique).

${ }^{17}$ Walter Koch, « Paläographie der Inschriften österreichischer Fresken bis 1530 », Mitteilungen des Instituts für Osterreichische Geschichtsforschung, t. 77, 1969, p. 1-42.

${ }^{18}$ G. Batelli, Lezioni di paleografza, Cité du Vatican, 2e éd., 1939 ; Mallon, op. cit., p. 21.

${ }^{19}$ F. Grossi Gondi, op. cit., p. 176-177.

${ }^{20}$ P. Deschamps, op. cit., p. 9.

${ }^{21}$ J. Mallon, op. cit., p. 22-23.
} 
siècle d'onciales fermées. Sont particulières à Vienne les formes suivantes $\{\xi$. Vienne a deux exemples, en 1226 et 1239, de ces lettres « à étranglement » qui sont représentées surtout à Toulouse et

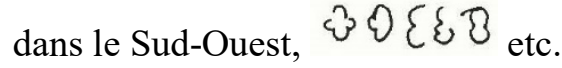

À Toulouse la réglure à deux traits pour chaque ligne, connue au moins dès le $\mathrm{XI}^{\mathrm{e}}$ siècle, devient habituelle à partir de 1152. La ponctuation par trois points verticaux entre chaque mot devient générale à partir de la fin du XII ${ }^{\mathrm{e}}$ siècle. On emploie des $O$ suscrits dans la datation à partir de 1167. Les lettres carrées se maintiennent jusqu'en 1230, inscription au caractère très archaïque. Toulouse ignore presque totalement lettres perlées et lettres fleuries, et emploie fort peu le redoublement de trait. Les onciales se ferment dès 1223 , et deviennent de règle au cours de la seconde moitié du siècle. Les lettres " à étranglement » sont particulièrement nombreuses et variées en 1104 ou environ, puis à compter de laseconde moitié du XII ${ }^{\mathrm{e}}$ siècle. Les caractères sont très proches à Narbonne, si ce n'est que redoublement du trait, lettres perlées, lettres fleuries, y sont un peu moins rares, les lettres «à étranglement $»$ au contraire nettement moins nombreuses ; les onciales se ferment à partir de 1257 .

Je crois que c'est la multiplication des études détaillées, pour des régions données, qui permettra un réel progrès dans les domaines de l'écriture des inscriptions. Il faudra veiller à mettre à part l'exemple isolé et bien souligner les temps forts et les continuités.

La cinquième observation concerne la nomenclature des écritures et la terminologie. On a publié, à l'occasion du premier congrès international de paléographie latine tenu à Paris en 1953, une Nomenclature des écritures livresques du IX $X^{e}$ s. au $X V I^{e}$ siècle, qui juxtapose plusieurs rapports tout en soulignant « la difficulté d'arriver à un accord unique, valable pour toutes les écritures latines employées jusqu'au $\mathrm{XV}^{\mathrm{e}}$ siècle inclus dans les livres manuscrits ${ }^{22}$. On constate la même difficulté pour la paléographie des chartes. La première préoccupation, elle réalisable, devrait être de relever les termes techniques employés dans les études épigraphiques réalisées jusqu'ici, et de définir pour chaque pays une terminologie simple. On pourra, ensuite, confronter les vocables propres à la discipline, et peut-être aboutir à des définitions communes pour certains d'entre eux, sans prétendre nécessairement à un lexique commun international. Une fois la terminologie exactement posée, les études mêmes de l'écriture donneront des indications sur la chronologie de son évolution. Alors, mais alors seulement, on pourra reposer la question d'une nomenclature, sans être assuré pour autant qu'une terminologie commune soit possible. L'essentiel est que chaque équipe d'épigraphistes établisse un vocabulaire clair et simple, auquel tout chercheur aura un accès facile. On a posé la question d'un parallélisme entre l'évolution de l'écriture épigraphique - pour laquelle on trouve parfois les termes de "romane » ou « gothique » - et l'évolution de l'histoire de l'art. Il y a des rapprochements possibles, mais plus utiles pour une présentation simplifiée dans un cadre pédagogique que solides au point de vue de l'argumentation scientifique. Bernhard Bischoff a écrit à ce sujet: "Est-il possible de retrouver les divisions de l'histoire de l'art dans le développement de l'écriture ? Cela me paraît, malgré toute la variété extérieure des formes, extrêmement difficile, parce que les périodes d'art et d'écriture ne coïncident pas toujours $\gg{ }^{23}$.

\section{Les supports}

L'étude des inscriptions antérieures à 1300 amène à considérer les supports les plus variés. La géologie explique que l'on trouve des inscriptions sur ardoise en Anjou ou dans les régions les plus voisines, du granit dans le Massif central, du marbre au sud d'une ligne de Bordeaux à Vienne, exception faite d'un autel portatif à la Souterraine (Creuse). On peut aussi souligner deux traits généraux portant l'un sur la chronologie, l'autre sur la topographie. Lorsque l'on passe de l'art roman à l'art gothique aux $\mathrm{XII}^{\mathrm{e}}-\mathrm{XIII}{ }^{\mathrm{e}}$ siècles, la peinture murale cesse d'être un des moyens de choix de décor des églises ; les murs sont percés de baies de plus en plus importantes, et le décor se reporte sur le vitrail dans le chœur et les parties hautes de l'église, sur les tapisseries dans les parties

\footnotetext{
${ }^{22}$ Paris, 1954 (Colloques internationaux du Centre National de la Recherche scientifique. Sciences humaines, IV).

${ }^{23}$ Ibid., p. 8.
} 
basses. Les représentations imagées se font rares sur les chapiteaux lorsque la statue devient quasi indépendante du mur. L'objet d'orfèvrerie est fabriqué dans des ateliers, à partir de modèles devenus conventionnels, les grands théologiens sont peu nombreux après Thomas d'Aquin, et l'inscription aura tendance à devenir plus banale, voire à disparaître, sur bien des émaux, des sculptures, de reliquaires ou autres objets sacrés, dans une iconographie bien fixée, qui ne demande plus de commentaire. Dans les épitaphes on emploiera de plus en plus, à partir des XII ${ }^{\mathrm{e}}-\mathrm{XIII}^{\mathrm{e}}$ siècles, l'effigie gravée ou le gisant. Bien des évolutions, finalement, s'observent à partir du XII ${ }^{\mathrm{e}}$ siècle et surtout du XIII ${ }^{\mathrm{e}}$ siècle, du fait de changement dans l'histoire de l'art ou dans l'histoire religieuse. Sur ce dernier point, par exemple, on notera que le report de l'autel au fond du chœur, avec messe dite dos au peuple, marque la disparition des devants d'autel, l'apparition des tabernacles, des retables. Les changements dans la pratique de l'eucharistie entraînent la création de fers à hostie ou d'ostensoirs. La fin du Moyen Âge verra le développement des tableaux peints dans les églises ou les monuments civils. L'autre trait général est la différence qui se marque entre France du Sud et France du Nord. La première est plus romane, la seconde plus gothique, autrement dit les peintures murales se trouvent plutôt au Sud, les vitraux plutôt au Nord. Le dynamisme économique sera plus marqué dans la France du Nord- Ile-de-France, Champagne, Picardie, Flandre... -, et c'est dans ces régions riches que l'on trouve davantage les objets d'art sacré précieux. Mais il y a aussi des différences qui traduisent des traditions, des sensibilités propres à chaque région. En parcourant le département de l'Yonne pour y étudier les inscriptions nous avons ainsi constaté que les épitaphes étaient plutôt sur de simples pierres au sud, dans la partie franchement bourguignonne, alorsqu'elles se trouvaient souvent sur des pierres tumulaires gravées de l'effigie du défunt ou autour des gisants, au nord, dans la partie du département qui touche la Champagne. Dans le tiers des départements de la France du Sud couverts par les publications du C.I.F.M. on a, avant 1300, une trentaine d'épitaphes accompagnant effigies gravées ou gisants ; dans le seul département de la Marne - en Champagne on en a quarante-deux.

\section{La métrique}

Bien d'autres évolutions marquent des changements à partir du XIII ${ }^{\mathrm{e}}$ siècle, et des différences entre France du Nord et France du Sud, qu'il s'agisse de la métrique, de la langue, des systèmes de datation, de l'iconographie, des formulaires, etc., car l'épigraphie apporte sa contribution à l'étude de la foi, de la culture du Moyen Âge, et elle enregistre les évolutions en ces domaines.

Les auteurs du Moyen Âge, lorsqu'ils veulent donner une inscriptions soignée, emploient volontiers la métrique dont on sait, par ailleurs, les vertus mnémotechniques. Ils se sont d'abord attachés aux modèles de l' Antiquité classique mais aussi aux modèles que leur offraient les poètes de!'Antiquité tardive ou du haut Moyen Âge, Sedulius, Juvencus, Prudence, Arator, Fortunat. Il s'agit naturellement de vers construits à partir de la quantité de syllabes. " Le vers le plus courant et le plus important, même au Moyen Âge sera l'hexamètre dactylique. Certains poètes du Moyen Âge connaissent si bien les différentes lois réglant la construction del' hexamètre queleurs vers, s'ils ne sont pas rimés, se distinguent à peine de ceux de l'Antiquité $»^{24}$. Mais le vers quantitatif n'est plus, au Moyen Âge, qu'une construction savante, car dès le temps de saint Augustin la différence entre syllabes brèves et syllabes longues a totalement disparu. Depuis Bède on appellera rythmique la poésie nouvelle qui ne tient plus compte de la quantité ${ }^{25}$. La transition sera fortement marquée par le vers léonin qui applique la rime entre hémistiche et fin de vers, tout en respectant les règles de la quantité. Ce vers, qui n'est pas ignoré de l'Antiquité, aura un succès particulier du XII ${ }^{\mathrm{e}}$ au XIII ${ }^{\mathrm{e}}$ siècle. Mais alors la connaissance des règles de quantité disparaîtra, la langue française se développera dans les inscriptions, et la prosodie cessera d'être au goût du jour. On estimera même que la recherche de la rime oblige à travestir la pensée.

«Nus hom ne puet chançon de jeste dire

\footnotetext{
${ }^{24}$ Dag Norberg, Introduction à l'étude de la versification latine médiévale, Stockholm, 1958, p. 7 et 64 (Acta universitatis Stockholmiensis. Studia latina Stockholmiensia, V).

${ }^{25}$ Ibid., p. 87.
} 


\section{Que il ne mente, la ou li vers define »}

assure l'auteur de la Mort Aymeri de Narbonne ${ }^{26}$, et les traducteurs de la Chronique du Pseudo-Turpin disent de même, Nicolas de Senlis en 1202 : "Nus contes rimés n'est verais », ou Jean, avant 1206 : "Et por ce que rime se velt afeiter de moz conqueilliz hors de l'estoire, voust li quens que cist livres Just sanz rime $»^{27}$.

Sur un ensemble de 1579 inscriptions des VIII ${ }^{\mathrm{e}}$-XIII ${ }^{\mathrm{e}}$ siècles dans la France du Sud, on en a 185 qui sont en hexamètres ou pentamètres, soit $11,7 \%$. Les vers classiques latins sont quatre fois moins nombreux que les vers léonins, et se trouvent bien plus avant 1200 qu'au XIII ${ }^{\mathrm{e}}$ siècle, ainsi en Haute-Garonne où 7 des 8 exemples sont antérieurs à 1200 . Les deux seuls exemples que l'on puisse donner du vers léonin avant l'an mil, à Toulouse à la fin du VIII ${ }^{\mathrm{e}}$ siècle, à Cavaillon au $\mathrm{IX}^{\mathrm{e}}-\mathrm{ou}$ aussi bien au $\mathrm{X}^{\mathrm{e}}$ siècle - comportent nombre d'erreurs de quantité. On ne peut, à partir d'exemples isolés, proposer de trop larges périodes chronologiques pour les différents usages en épigraphie à partir d'exemples très précoces ou très tardifs qui seraient exceptionnels. Sans écarter absolument un emploi précoce, on peut donc dire que le vers léonin ne commence vraiment, en France du Sud, qu'au $\mathrm{XI}^{\mathrm{e}}$ siècle. Le vers léonin simple s'y arrête souvent au XII ${ }^{\mathrm{e}}$ siècle et ne dépasse qu'exceptionnellement 1236 : un vers sur douze à La Sauve-Majeure en 1252, trois vers sur treize à Cavaillon en 1261, deux vers sur cinq à Brive en 1280, quatre vers sur six à Flassans (provenant des Cordeliers de Perpignan). Le vers léonin riche est constamment plus fréquent que le vers léonin simple. Sa grande période est le XII ${ }^{\mathrm{e}}$ siècle et il continue à être utilisé pendant tout le $\mathrm{XIII}{ }^{\mathrm{e}}$ siècle, mais son emploi va dès lors en décroissant : en Haute-Garonne deux, inscriptions du XI $\mathrm{XI}^{\mathrm{e}}$, quatorze du $\mathrm{XII}^{\mathrm{e}}$ siècle, trois du XIII ${ }^{\mathrm{e}}$ siècle, en Isère quatre du $\mathrm{XI}^{\mathrm{e}}$, dix du XII ${ }^{\mathrm{e}}$, sept du XIII ${ }^{\mathrm{e}}$. Le Roussillon fait exception, avec six exemples pour le XIIe siècle et quatorze pour le XIIIe siècle ${ }^{28}$. Encore doit-on noter que les derniers exemples, en 1280 et 1302 à Elne, à Céret en 1283, sont des emprunts à des formulaires métriques plus anciens ${ }^{29}$. Il faudra étudier de près les inscriptions d'Espagne où le vers léonin a été introduit tardivement fin $\mathrm{XI}^{\mathrm{e}}$-début $\mathrm{XII}^{\mathrm{e}} \mathrm{s}$. sous l'influence française, et où semble-t-il, il a été employé plus tard qu'en France. Le vers léonin riche est exceptionnel en France du Sud au XIV $^{\text {e }}$ siècle : deux exemples en 1347, aux Dominicains et aux Franciscains de Toulouse, un exemple à Saint-Pierre de Vienne vers 1357. On a dans la même France du Sud dix-neuf exemples de vers à rimes communes, simples ou riches, généralement en finales par distiques, cinq pour le XII ${ }^{\mathrm{e}}$, à partir de 1148, quatorze pour le XIII ${ }^{\mathrm{e}}$ siècle.

Il faudra mener une étude semblable pour la France du Nord de la Loire. On rencontre là aux $\mathrm{X}^{\mathrm{e}}-\mathrm{XII}{ }^{\mathrm{e}}$ siècles, des écoles réputées, Reims, Chartres, Paris, Tours, Angers, Le Mans ; de là sont originaires quelques-uns des poètes les plus connus, Hildebert de Lavardin, Baudri de Bourgueil. Il faudra examiner si la métrique y a été plus en honneur dans les inscriptions de cette époque. D'un autre côté le français y est plus employé dans les inscriptions au XIII ${ }^{\mathrm{e}}$ siècle, et naturellement sa vogue condamne la métrique classique latine. Des sondages amènent à constater que le vers léonin y paraît peut-être plus tard qu'au Sud, mais se maintient plus longtemps, et que les exemples de vers rimant deux à deux en finale y sont bien plus nombreux : dix-sept exemples pour le seul département de l'Aisne, en regard des dix-neuf exemples de toute la France du Sud. Autrement dit le vers rythmique a dû y avoir beaucoup plus de succès que le vers quantitatif. Cette esquisse devra être affinée, par l'examen des exemples situés hors des grands fréquences. Une meilleure connaissance des citations et formulaires conduira aussi à écarter des exemples tardifs qui ne serait que des emprunts. On notera

\footnotetext{
${ }^{26}$ La mort Aymeri de Narbonne, chanson de geste, éd. J. Couraye du Parc, Paris, 1884, p. 132 (Société des anciens textes français).

${ }^{27}$ Gaston Paris, Depseudo-Turpino, Paris, 1865, p. 44 et 56 ;Diesogenannte poitevinische Uebersetzung des PseudoTurpin nach den Handschrifien mitgetheilt von Theodor Auracher, Halle, 1877, p. 6 (extr. de la Zeitschrift für romanische Philologie). Au XIV ${ }^{\mathrm{e}}$ s. encore Jean Le Bel, dans sa Chronique, éd. Jules Viard et Eugène Déprez, t. I, Paris, 1904 (Société d'Histoire de France), p. 1-13, met en garde contre ceux qui écrivent en vers : «Et sy a grand plenté de parolles controuvées et de redictes pour embelir la rime ».

${ }^{28}$ En y joignant une inscription des Cordeliers de Perpignan de 1292 conservée au château de la Rouvière, commune de Flassans (Var).

${ }^{29}$ L'exemple même des Cordeliers de Perpignan (à Flassans) en 1292 correspond à un emprunt au moins partiel à un formulaire antérieur.
} 
en outre, des reprises de la métrique classique à la fin du Moyen Âge, dans la ligne cette fois de l'imitation des anciens chère aux auteurs de la Renaissance. Il s'agit là d'une autre problématique.

\section{La langue vulgaire}

Tôt on a cessé de parler latin ${ }^{30}$, mais les clercs, qui avaient un quasi-monopole de l'écrit, ont longtemps continué à écrire en latin. Les inscriptions peuvent apporter une utile contribution à l'histoire de l'évolution du latin et aux débuts de la langue vulgaire dans l'écrit : noms de personne et de lieu que l'on laisse en langue vulgaire, grammaire qui se simplifie, construction qui cesse d'être latine alors que l'habit est encore latin, enfin premiers textes en français. Pour le domaine français trois inscriptions en langue vulgaire ont été relevées pour le $\mathrm{XII}^{\mathrm{e}}$ siècle, français au baptistère Saint-Jean de Poitiers ${ }^{31}$, occitan pour un émail de Grandmont au Musée de Cluny $^{32}$ et pour le nom d'un maître d'œuvre à Castillon- en-Couserans ${ }^{33}$.

Si l'on reprend l'exemple de la France du Sud, pour laquelle la base de données établie à partir des volumes publiés au C.E.S.C.M. permet une étude d'ensemble, on constate que $43 \%$ des inscriptions recensées entre la fin du VIII et 1300 portent sur le XIII ${ }^{\mathrm{e}}$ siècle. Dans les 685 inscriptions du XIII ${ }^{\mathrm{e}}$ s., le pourcentage des inscriptions en langue vulgaire est de 5,4\%, soit 40 textes. Sur ces 40 textes 33 sont de la seconde moitié du siècle ou datés seulement du siècle. Trois de ces textes sont en ancien français ${ }^{34}$, les autres sont en occitan. Ces textes sont nettement plus nombreux au Sud-Ouest (Toulouse, Cahors, Rocamadour, Limoges... ) qu'au Sud-Est (seulement quatre inscriptions en occitan à l'est du Rhône). Il est significatif qu'à Vienne les soixante-seize inscriptions du XIII ${ }^{\mathrm{e}}$ siècle soient toutes en latin. De façon générale les établissements religieux anciens sont fidèles au latin. Sur les 40 exemples relevés aucun ne vient à proprement parler d'une cathédrale ${ }^{35}$.

Des sondages dans le fichier général conservé à Poitiers montrent que la proportion d'inscriptions en français au XIII ${ }^{\mathrm{e}}$ siècle est beaucoup plus importante dans la France du Nord. A Châlons-sur-Marne sur cinquante inscriptions du XIII ${ }^{\mathrm{e}}$ siècle trente-huit sont en français, le premier exemple étant de 1223, à Reims sur dix-huit inscriptions du XIII ${ }^{\mathrm{e}}$, quatre seulement sont en français, dans la Somme six sur vingt - à compter de 1220 -, dans l'Aisne vingt-et-une sur soixante-dix - à partir de 1225 -, à Rouen quinze sur trente-huit - à partir de 1237 -, à Dijon huit sur trente-quatre, dans l'Yonne trente-sept sur cent vingt-huit, dans l'Aube onze sur quarante-trois. La majeure partie de ces textes en langue vulgaire se trouvent chez les ordres mendiants ou dans des abbayes déterminées, Notre-Dame-en-Vaux à Châlons, Longpont dans l'Aisne, Saint-Ouen à Rouen, mais pas dans les cathédrales. À Châlons ce sont toutes les inscriptions chez les Franciscains, et presque toutes celles chez les Dominicains qui sont en langue vulgaire.

L'évolution est sans doute assez comparable en Espagne, où dès la seconde moitié du XIII siècle les inscriptions en langue vulgaire vont se multiplier ${ }^{36}$. Il me semble que les débuts de la langue vulgaire dans les inscriptions sont plus tardifs en Italie, Allemagne, Pologne. On sait l'existence d'une tradition anglo-saxonne dans les inscriptions des îles britanniques. Il ne faut sûrement pas

\footnotetext{
${ }^{30}$ Dag Norberg, " A quelle époque a-t-on cessé de parler latin en Gaule ? ", Annales. Économies. Sociétés. Civilisations, XXI, 1966, p. 346-356 (l'auteur estime que les changements importants se généralisent au Viles.).

${ }^{31}$ C.I.F.M., 1, Ville de Poitiers, éd. R. Favreau, J. Michaud, Paris et Poitiers, 1974, n ${ }^{\circ} 11$, p. 12.

${ }^{32}$ Geneviève Souchal, «Les émaux de Grandmont au XII ${ }^{e}$ siècle », Bulletin monumental CXX, 1962, p. 339-357.

${ }^{33}$ C.I.F.M., 8, Ariège, Haute-Garonne, Haute-Pyrénées, Tarn-et-Garonne, éd. R. Favreau, J. Michaud, B. Leplant, Paris, 1982, Ariège, n $^{\circ} 1$, p. 7.

${ }^{34}$ A Bordeaux, Castres, Panjas.

${ }^{35}$ L'inscription de 1203 à la cathédrale d'Elne vient du prieuré de cisterciennes de l'Eule ; l'inscription $\mathrm{du}$ XIII ${ }^{\mathrm{e}}$ siècle au trésor de la cathédrale est sur un anneau épiscopal et signale des reliques.

${ }^{36}$ On peut l'observer dans la thèse soutenue par Maximino Guttierez Alvarez sur Las inscripciones medievales de la provincia de Zamora à l'Université de Léon en 1991 sous la direction du professeur Vicente Garcia Lobo, p. LVI et Catalogue général (premier texte sûr en 1258. En la province de Burgos l'inscription de Rebolledo de la Torre de 1186 est déjà très proche de la langue vulgaire : QUANDO POBLADO FUIT ISTUM SOLAR (José Perez Carmuna, Arquitectura y escultura romanicas en la provincia de Burgos, Burgos, 1959, p. 41-44).
} 
généraliser les études faites à partir d'une région donnée, comme le montred'ailleurs l'exemple de la France du Sud par rapport à la France du Nord.

\section{Datation}

La façon de dater montre aussi et les changements qui se marquent au XIII ${ }^{\mathrm{e}}$ siècle, et les différences d'évolution que l'on peut observer selon les régions, et en particulier entre France du Sud et France du Nord.

La datation à partir des années de l'Incarnation s'observe à partir du $\mathrm{IX}^{\mathrm{e}}$ siècle en France et en Italie, mais ne s'affirme vraiment qu'à compter du $\mathrm{XI}^{\mathrm{e}}$ siècle, et ne se rencontre dans nombre de départements qu'à partir du XII ${ }^{e}$ ou même du XIII ${ }^{e}$ siècle. Pour préciser le jour du mois l'emploi prolongé du calendrier romain montre bien le conservatisme des usages en épigraphie et l'imprégnation de la culture romaine. Conservatisme car on le trouve encore dans les inscriptions longtemps après qu'il ait été abandonné dans les chartes, par exemple en Bourgogne et Roussillon où il sera utilisé jusqu'au $\mathrm{XV}^{\mathrm{e}}$ siècle. Dans la France du Sud son emploi est plus précoce que dans la France du Nord mais en même temps il s'arrêtera plus tôt : dans les pays au sud de la Loire et du Rhône on ne le trouve plus après 1200 que dans une dizaine de départements ; en même temps l'utilisation du calendrier romain est nettement plus fréquent au sud qu'au nord : quinze départements au sud d'une ligne Nantes-Colmar ont plus de vingt exemples - et jusqu'à plus de cent trente-, alors qu'au nord de cette ligne on ne trouve cette vingtaine d'exemples que dans la région parisienne prise dans son ensemble et en Pas-de-Calais. L'emploi du quantième du mois à la façon moderne apparaît au $\mathrm{XI}^{\mathrm{e}} \mathrm{s}$., se généralise à partir de la seconde moitié du XIII ${ }^{\mathrm{e}}$ siècle.

La datation à partir d'une fête religieuse est très rare avant la seconde moitié du XII ${ }^{\mathrm{e}}$ siècle, et se généralise à partir du troisième tiers du $\mathrm{XIII}^{\mathrm{e}}$ siècle. De façon très nette on la trouve employée principalement de la Normandie à la Bourgogne, alors qu'elle est très peu utilisée dans la France du Sud ; quinze des dix-sept départements où on ne la rencontre pas du tout sont d'ailleurs au sud d'une ligne allant des Landes à laSavoie ${ }^{37}$.

\section{Culture, foi, mentalités}

Les inscriptions permettent encore de suivre l'évolution de la culture, de la foi, des mentalités, des attitudes sociales. Là aussi des changements forts s'observent à partir des $\mathrm{XII}^{\mathrm{e}}-\mathrm{XIII}{ }^{\mathrm{e}}$ siècles. Les pouvoirs à prétention universelle, papauté et empire, connaissaient un arrêt devant l'affirmation progressive des États de caractère modernes, Angleterre et France principalement. Le monde rural cède le pas devant l'apparition de nouveaux réseaux urbains, de communes, d'une classe « bourgeoise ». Les grandes écoles capitulaires et monastiques disparaissent après la création des universités. Le latin cesse d'être l'unique langue de culture devant l'éclosion et l'épanouissement des littératures en langue vulgaire. Les grands écrits théologiques se font rares après l'Euvre magistrale de Thomas d'Aquin. L'art gothique fait suite à l'art roman. L'individualisme s'affirme.

Il ne saurait être question de traiter sur le fond de cette question. Quelques exemples suggèrent combien ces changements sont marquants et traduisent, dans l'ensemble du Moyen Âge, le passage à une phase nouvelle qui préludera à la Renaissance et aux temps modernes.

Dans les épitaphes des $\mathrm{IX}^{\mathrm{e}}-\mathrm{XIII}{ }^{\mathrm{e}}$ siècles fréquentes sont les mentions d'une noble origine, avgc les mots strips, gens, genus, progenies, semen, stemma, sanguis, germen, radix ou nobilis, noфilitas, nobiliter, illustris, etc. À partir du XIII ${ }^{\mathrm{e}}$ va se développer une grandiloquente titulature qui ne fera que s'amplifier avec les siècles suivants : « haute et puissante dame » (1261), " très haute dame madame ... » (1271), « très haut et très loyal chevalier » (1271), « très haut et puissant prihce » (1271), tandis que d'un autre côté on souligne désormais avec le plus grand soin les grades universitaires. Déjà au XII ${ }^{\mathrm{e}}$ siècle la qualité des grands intellectuels était vivement soulignée : " celeberrimus iste magister » dit Marbode pour Anselme de Laon (1117), "magister celeberrimus

\footnotetext{
${ }^{37}$ Étude à paraitre dans les actes du colloque d'épigraphie tenu à Rome en septembre 1993.
} 
Parisiensis » proclame l'épitaphe de Joscelin, évêque de Soissons (1151), " Gallorum Socrates, Plato maximus. ., noster Aristoteles... » pour Pierre Abélard (1142). À partir du XIII ${ }^{\mathrm{e}}$ s. on donnera du " magister " à ceux qui sont passés par l'Université et on indiquera les degrés qu'ils ont obtenus, "licenciatus in decretis », " in legibus licentiatus », " in medicina doctor », " in artibus magistri et in legibus licentiati » par exemple pour des chanoines de Saint-Hilaire-le-Grand de Poitiers au XV siècle. Dans les épitaphes des $\mathrm{IX}^{\mathrm{e}}-\mathrm{XII}{ }^{\mathrm{e}}$ siècles on fait principalement état des qualités morales du défunt : le doyen de Saint-Hilaire-le Grand de Poitiers mort à la fin du $\mathrm{X}^{\mathrm{e}}$ siècle est dit pacificus, Domini plenus amore sui, corporeo vultu jocundus, mente benignus, pauperibus clemens, justitiam sitiens, aecclesiae cultor, utilisin multibus fratribus ${ }^{38}$. Dès le XIII ${ }^{\mathrm{e}}$ siècle, mais de plus en plus aux $\mathrm{XIV}^{\mathrm{e}}$ et $\mathrm{XV}^{\mathrm{e}}$ siècles, on donne les charges du défunt, on déroule tout un « cursus », en terminant ordinairement, - même pas toujours - par une brève demande de prière. En 1282 meurt à Toulouse, " illustrissimo Philippa rege Francorum, reverendissimo et valentissimo Bertrando episcopo Tholosano. Magister Aymericus, canonicus, cancellarius et operarius ecclesie Tholosane, cujus anima requiescat in pace, amen ${ }^{39}$. L'épitaphe d'un haut fonctionnaire du royaume aux Cordeliers de Poitiers dit : "Cy devant gist noble homme et sage Andry Marchant, lequel en son vivant fut conseiller du feu roy Charles VI et du roy Charles VII qui est à présent en leur court de Parlement, et depuis fut conseiller et chambellan desdiz roys et en son temps successivement bailli de Sens et d'Auxerre, bailli de Meux, prévost de Paris, gouverneur du duchiéet capitaine de la ville d'Orléans pour monseigneur le duc d'Orléans, lequel trespassa en ceste villelejour Sainte Anne l'an milquatrecens trente et neuf $\rangle^{40}$.

Dans les épitaphes des $\mathrm{IX}^{\mathrm{e}}$-XII ${ }^{\mathrm{e}}$ siècles l'apostrophe au lecteur, la demande de prières pour le défunt sont ordinaires. À partir essentiellement du XIII ${ }^{\mathrm{e}}$ siècle on va fonder des services anniversaires, des messes, des chapelles, faire des legs à différents ecclésiastiques à charge de prières, afin de mieux se trouver protégé, pense-t-on, lorsqu'il faudra comparaître devant le Christ juge. Il y a là une piété « calculatrice », " arithmétique », qui marque un esprit plus individualiste et une crainte plus forte du jugement. Les inscriptions en sont un reflet fidèle, où les exemples sont innombrables. Sur 120 exemples du mot anniversarium avant 1300, 116 sont du XIII ${ }^{\mathrm{e}}, 3 \mathrm{du} \mathrm{XII}{ }^{\mathrm{e}}, 1$ au XI ${ }^{\mathrm{e}}$ siècle ; parfois on emploie un autre mot, ainsi pour des fondations de " repas » à l'intention des clercs qui célébreront des prières chaque année pour le défunt, le terme de refectio, à quinze reprises, à Vienne. On rejoint la même crainte de l'au-delà avec les indulgences que l'Église distribue, à partir du $\mathrm{XI}^{\mathrm{e}}$ siècle, à ceux qui partent à la croisade, qui assistent aux anniversaires de la dédicace d'une église, qui font des donations aux églises, qui participent aux jubilés institués en 1300, etc. Dans les inscriptions on en trouve de premiers exemples à la fin du $\mathrm{XI}^{\mathrm{e}}$ et au XII ${ }^{\mathrm{e}}$ siècle en Italie (une douzaine d'inscriptions du $\mathrm{XII}^{\mathrm{e}}$ ), en 1166 à Nogal de las Huertas en Espagne, à partir de 1200 en France, en 1235 à Newenham en Angleterre, etc.

Dans le domaine des commentaires épigraphiques de l'iconographie on note aussi des changements vers le XIII ${ }^{\mathrm{e}}$ siècle. Dans les textes qui accompagnent l'image au XI ${ }^{\mathrm{e}}$, au XII ${ }^{\mathrm{e}}$ et encore en partie au XIII ${ }^{\mathrm{e}}$ siècle, au moins dans certains pays, il y a des développements, souvent fort beaux, tirés de la Bible, de la liturgie, des auteurs chrétiens qui pendant des siècles ont pratiqué une exégèse historique, allégorique ou typologique, morale ou tropologique, et enfin anagogique, pour reprendre les termes du beau livre d'Henri de Lubac ${ }^{41}$. Que l'on pense par exemple à l'iconographie du retable de Nicolas de Verdun à Klosterneuburg, aux vitraux de l'abbé Suger à Saint-Denis, ou aux fonts baptismaux de la cathédrale d'Hildesheim ${ }^{42}$. Peu à peu ce type de commentaire disparait dans le domaine épigraphique. Fréquentes sont au XII ${ }^{\mathrm{e}}$ siècle les représentations de la Vierge à l'Enfant, avec un caractère hiératique, la Mère présentant son Fils qui est Dieu:

\footnotetext{
${ }^{38}$ C.I.F.M., 1, Ville de Poitiers, éd. R. Favreau, J. Michaud, Poitiers et Paris, 1974, n 59, p. 59-61.

${ }^{39}$ C.I.F.M., 7, Ville de Toulouse, éd. R. Favreau, J. Michaud, B. Leplant, Paris, 1982, n 95, p.135.

${ }^{40}$ Longuemar, «Épigraphie du Haut Poitou », Mém. Soc. Antiq. Ouest, t. XXVIII, 1863, n 119, p. 239 : l'épitaphe est conservée au Musée de la ville de Poitiers.

${ }^{41}$ Henri de Lubac, S.J., Exégèse médiévale. Les quatre sens de l'Écriture, Paris, 3 vol., 1959-1961 (Théologie. Études publiées sous la direction de la Faculté de Théologie S.J. de Lyon-Fourvière, 41-42).

42 Sur ce problème, R. Favreau, « L'apport des inscriptions à la compréhension des programmes iconographiques », Lecturas de Historia del Arte. Ephialte, Vitoria-Gasteiz, 1992, p. 33-50.
} 
"In gremio Matris residet sapientia Patris » ${ }^{43}$, "Stella parens salis, cultores dirige prolis » (Lyon, Musée, XII ${ }^{\mathrm{e}}$ s.), " Stella parit solem, rosaflorem, forma decorem »(Palerme, chapelle palatine), "Virgo Deum complexa sinu servando pudorem, virgineum matris fundans per secula nomen »(Rome, S. Maria in Trastevere), etc. Avec le XIII ${ }^{\mathrm{e}}$ siècle on verra des statues de jeune mère se déhanchant pour tenir l'Enfant, ou même Marie seule, et la beauté de la représentation semble alors renvoyer au second plan l'affirmation capitale du concile d'Ephèse : Marie Mère de Dieu. Les inscriptions du même coup disparaissent, d'autant plus qu'elles ne sont pas nécessaires pour identifier la scène. De même les Jugements derniers de tympans romans, les représentations romanes du Christ en Majesté, sont fréquemment accompagnées de commentaires par des inscriptions aux $\mathrm{XI}^{\mathrm{e}}$ et $\mathrm{XII}^{\mathrm{e}}$ siècles. Ce commentaire disparaît par la suite ou du moins se fait rare. Longtemps on identifie les saints par leur nom, on précise leurs fonctions, le rôle qu' on leur attribue. Puis l'iconographie se fixe, les attributs se multiplient, et il n'est plus nécessaire d'avoir un accompagnement épigraphique.

Dans le domaine même de la foi l'épigraphie apporte son complément d'information. Il n'est pas en Occident, comme en Orient, de grandes querelles théologiques, mais chacun sait combien l'enseignement de Bérenger de Tours au $\mathrm{XI}^{\mathrm{e}}$ siècle a conduit à un approfondissement théologique du sacrement de l'eucharistie, à une définition précise de la " transsubstantiation », le débat conduisant à un développement marqué du culte du « Saint Sacrement » à partir du XIII ${ }^{\mathrm{e}}$ siècle. Les inscriptions sur les calices, les patènes et autres objets de culte reflètent ce débat sur ce sacrement.

À partir de la centaine d'inscriptions réunie en un premier dossier, on peut faire une histoire du sacrement: la croyance des $I^{\mathrm{e}}-\mathrm{X}^{\mathrm{e}}$ siècles, les débats sur la présence réelle qui font suite aux affirmations de Bérenger, les figures de l'Ancien Testament, l'approfondissement théologique des $\mathrm{XII}^{\mathrm{e}}$-XIII ${ }^{\mathrm{e}}$ siècles, l'institution de la Fête-Dieu et le développement hymnographique, etc. Sur le calice d'Essen- Werden dit de saint Liudger, de la seconde moitié du $\mathrm{IX}^{\mathrm{e}}$ ou du $\mathrm{X}^{\mathrm{e}}$ siècle on a gravé simplement : HIC CALIX SANGUINIS DOMINI NOSCTRI IHESU CHRISTI ${ }^{44}$.

Après Bérenger on insiste sur la présence réelle et on met en garde contre les déviations :

\section{CARMEN QUAM GUSATS NON ATTERIT ULLA VETUSTAS}

PERPETUUS CIBUS [QUI]NEGAT HOC REUS [EST] AMEN,

sur la patène de l'abbé Pélage au Louvre ${ }^{45}$, ou

CONSTAT IN ALTARI CARMEN DE PANE CREARI

DA DEUS IN REBUS QUOD SUMITUR IN SPECIEBUS

sur la patène de Fritzlar. Sur la patène de Trzemeszeno, au trésor de la cathédrale de Gniezno dix scènes de l'Ancien Testament sont données comme des figures du Christ : CLAMANT SCRIPTURE QUOD SIGNAVERE FIGURE ${ }^{46}$. L'iconographie et son accompagnement épigraphique renvoient à la fois à l'Ancien et au Nouveau testament sur le calice et la patène de Wilten au Kunsthistorisches Museum de Vienne ${ }^{47}$, et l'ensemble des textes sont d'une grande richesse aux XII ${ }^{\mathrm{e}}$ et $\mathrm{XIII}^{\mathrm{e}}$ siècles, mais il semble bien, là aussi, que le commentaire épigraphique s'affadisse ou même disparaisse souvent dans les siècles qui suivent.

\footnotetext{
${ }^{43}$ R. Favreau, «Origines et succès d'une formule épigraphique : In gremio Matris residet Sapientia Patris », Disœrnere vera ac falsa [Mélanges Josef Szymanski], Dublin, 1992, p. 99-108 (Annales Universitatis Marie Curie-Sktodowka, XLV, 1990).

${ }^{44}$ Victor H. Elbern, " Der eucharistische Kelch im frühen Mittelalter », Zeitschrift des deutschen Vereins für Kunstwissenschaft, XVII, 1-2, 1963, n 9, p. 69.

${ }^{45}$ R. Favreau, "Les inscriptions du calice et de la patène de l'abbé Pélage au Louvre », Comptes rendus de l'Académie des Inscriptions et Belles Lettres, 1993, p. 31-48.

46 Piotr Skubiszewski, "Die Bildprogramme der romanischen Kelche und Patenen », Metallkunst von der Spatantike bis zum ausgehenden Mittelalter, dir. Arne Effenberger, Berlin, 1982, p. 202-203.

${ }^{47}$ Ibid., p. 213-219.
} 
Les inscriptions antérieures au XIII ${ }^{\mathrm{e}}$ siècle appellent une problématique qui a ses caractères propres. C'est vrai sur des aspects qui touche la forme comme l'écriture, les différents types de supports, l'emploi de la métrique, la langue. C'est vrai plus encore, et plus profondément, pour l'étude du contenu des textes. Le passage d'une culture cléricale, qui a un quasi-monopole jusqu'au $\mathrm{XII}^{\mathrm{e}}$ siècle, à une culture plus ouverte au temps des universités, de la montée en puissance des villes, du développement d'États de type «moderne», avec dans le même temps l'arrêt des grandes fondations monastiques et les graves difficultés de l'Église aux $\mathrm{XIV}^{\mathrm{e}}$ et $\mathrm{XV}^{\mathrm{e}}$ siècles, ne pouvait qu'entraîner des changements marqués sur le fond des inscriptions.

Avant 1300 plus qu'après l'épigraphiste devra avoir recours à la théologie, l'exégèse, la liturgie, l'hymnologie, l'Écriture, et de façon générale à l'ensemble des auteurs de l'Antiquité tardive aux XII $-X I I I^{\mathrm{e}}$ siècles. Et comme il s'agit d'une culture généralement répandue dans l'ensemble de la chrétienté, il ne pourra se limiter à l'étude des inscriptions dans un cadre géographique trop étroit. En fin de compte la date de 1300 qui a été imposée à l'équipe française lors de sa création n'est pas une absurdité. Mais il serait assurément absurde de considérer cette date comme une rupture, un arrêt. Les changements qui s'observent au XIII ${ }^{\mathrm{e}} \mathrm{s}$. ne peuvent être isolés des siècles qui suivent. Souligner les différences n'exclut pas les continuités qui sont si évidentes, par exemple, dans le formulaire, et l'Histoire n'a que faire de ces limites chronologiques que nous imposent les évidentes contraintes matérielles de personnel et d'argent, et la simple nécessité pratique de fixer des étapes dans une recherche historique. 